pre-treatment with CGRP or SP in normal control rats were comparable to those of IPC. In addition, inhibition of CGRP or SP receptor essentially abolished the cardiac protective effects of IPC in normal control rats, but not in diabetic rats. IPC resulted in significant increase of CGRP and SP release in coronary effluent of normal control hearts, and which were effectively inhibited by TRPV1 receptor inhibitor, capsazepine or ruthenium red. However, IPC had no effects on CGRP and SP release in coronary effluent of diabetic hearts in the presence or absence of capsazepine or ruthenium red. Conclusions Cardioprotection by IPC against ischaemia/reperfusion injury is lost during diabetes, and their underlying mechanism is partly associated with the decreased CGRP and SP release due to the impairment of TRPV1 receptor in diabetic hearts.

\section{e0064 MICRO RNAS ARE INVOLVED IN THE PSYCHOLOGICAL STRESS-INDUCED CARDIAC DISORDERS IN RATS}

doi:10.1136/hrt.2010.208967.64

Liu Bin, Wang Yuehui, Wang Lishuang, Zhao Zhuo, Wang Xiaodong. The Second Hospital of Jilin University

Objective Psychological stress has become an important factor in the development of cardiac disorders. MicroRNAs (miRNA) have been implicated in regulation of cardiovascular diseases. Therefore, we investigated whether miRNAs are involved in the psychological stress-induced cardiac disorders

Methods Stress rat models were established by complex stimulation at different times during the daytime for 2 weeks. Body weight, blood pressure and ECG were measured) every 3 days during processing stress. Adrenocorticotrophic hormone (ACTH) was measured by using ELISA. Cardiac changes were detected by HE staining and electronic microscopy. MicroRNA microarray was used for analysing the differential expression of miRNAs.

Results After psychological stress, rats displayed change in behaviour. Body weight increased slowly and systolic blood pressure increased significantly in stress group from the 6 th day to the 15 th day. The ECG of all rats was normal before experiment. 2 weeks after stimulation the ECG record of different individuals in stress group showed different arrhythmia, including sinus tachycardia, atrial premature contraction, ventricular arrhythmia, and ST-T changes. Hypothalamic-pituitaryadrenal activity increased in stress group compared to control group by detecting ACTH. And the ultrastructure and histology showed injury changes in stress group. Compared to the control group, there were 55 different miRNAs in stress model including upregulation of 20 and downregulation of left, among which miRNA-141, miRNA-382, miRNA-219-5p and miRNA-296 up-regulate, miRNA-135a and miRNA-466b are significant down-regulate.

Conclusions Complex stimulation can induce psychological stress, which can cause cardiac injury. MiRNAs change in stress rat models, including upregulation of miR-141, miR-382, miR-219-5p and miR296 , and significant downregulation of $\mathrm{miR}-135 \mathrm{a}$ and $\mathrm{miR}-466 \mathrm{~b}$, which may play important roles in psychological stress-induced cardiac disorders. (Supported by research grant NSFC 30940041).

\section{e0065 THE ASSOCIATION BETWEEN THE SINGLE NUCLEOTIDE POLYMORPHISMS OF MATRIX METALLOPROTEINASES AND THE CAROTID ATHEROSCLEROSIS IN PATIENTS WITH ESSENTIAL HYPERTENSION}

doi:10.1136/hrt.2010.208967.65

${ }^{1}$ Yan-Ping Ma, ${ }^{2}$ Xin-Juan Xu, ${ }^{2}$ Fang Zhao, ${ }^{2}$ Guang-Mei Hu, ${ }^{2}$ Jun-Shi Zhang. ${ }^{1}$ Department of Hypertension, The First Affiliated Hospital of Medical Univercity, Urumchi
Xinjiang, China; ${ }^{2}$ Department of Hypertension, The Xinjiang Armed Police Hospital, Urumchi Xinjiang, China

Objective To investigate the relationship between the single nucleotide polymorphisms (SNP) of matrix metallo proteases (MMP-2 -735C/T; MMP-3 -1171 5A/6A) and the carotid atherosclerosis (CAS) in Chinese Han and Uygur populations with EH.

Methods The study comprised 276 Han nationality and 212 Uygur participants, who were divided into two groups: CAS $(n=293)$ and NS $(n=195)$. Genotypes were detected by PCR-RFLP and their frequencies were determined.

Results (1) The frequencies of MMP-2 TT genotype and T allele in CAS were higher than in NS (Han : $X^{2}=11.441, p=0.003$; Uygur $\left.\mathrm{X}^{2}=28.255, \mathrm{p}=0.000\right)$. In NS, the frequencies of TT genotype and T allele in Han were higher than in Uygur $\left.\left(X^{2}=12.509, p=0.001\right)\right)$. (2) The frequencies of MMP-3 6A/6A genotype and 6A allele in CAS were higher than NS (Han: $X^{2}=7.523, p=0.024$; Uygur: $X^{2}=6.474$, $\mathrm{p}=0.039)$. The frequencies of $\mathrm{MMP}-36 \mathrm{~A} / 6 \mathrm{~A}$ genotype and $6 \mathrm{~A}$ allele in Han were higher than Uygur (CAS: $X^{2}=26.230, p=0.000$; NS: $\left.X^{2}=18.809, p=0.000\right)$. (3) The single gene analysis showed Han individuals with CT or TT genotypes had 2.25-fold risk and Han individuals with $6 \mathrm{~A} / 6 \mathrm{~A}$ genotypes had 1.85 -fold risk suffering from CAS. Han individuals with both T allele and $6 \mathrm{~A} / 6 \mathrm{~A}$ genotypes had 3.17-fold risk suffering from CAS. The single gene analysis showed that Uygur individuals with CT or TT genotypes had 5.04-fold risk suffering from CAS. Uygur individuals with 6A/6A genotypes had 2.20-fold risk suffering from CAS. Uygur individuals with both $\mathrm{T}$ allele and 6A/6A genotypes had 3.20-fold risk suffering from CAS. (4)According to MMP-2 genetypes, Han individuals with MMP-2 CT + TT genotypes had higher LDL and lower HDL levels than CC genotype in CAS (LDL:2.9 mmol/l vs $2.6 \mathrm{mmol} / \mathrm{l}$; HDL:1.2 mmol/1 vs $\mathrm{mmol} / \mathrm{l}$ ). Uygur individuals with CT + TT genotypes had higher TG levels than CC genotype (CAS : $2.5 \mathrm{mmol} / \mathrm{l}$ vs $1.6 \mathrm{mmol} / \mathrm{l}$; NS : $3.9 \mathrm{mmol} / \mathrm{l}$ vs $2.0 \mathrm{mmol} / \mathrm{l}$ ). According to $\mathrm{MMP}-3$, Han individuals with $6 \mathrm{~A} / 6 \mathrm{~A}$ genotype had higher T-CHOL and LDL levels than $5 \mathrm{~A} /$ $5 \mathrm{~A}+5 \mathrm{~A} / 6 \mathrm{~A}$ genotypes in NS group (T-CHOL : $4.6 \mathrm{mmol} / \mathrm{l}$ vs $4.2 \mathrm{mmol} / \mathrm{l}$; LDL: $2.3 \mathrm{mmol} / \mathrm{l}$ vs $2.2 \mathrm{mmol} / \mathrm{l}$ ). (5) The binary logistic regression analysis showed MMP-2 CT + TT genetypes were the risk factors for CAS in individuals with $\mathrm{EH}$ (Uygur: $\mathrm{OR}=9.65$; Han : $\mathrm{OR}=2.076$ ). MMP-3 6A homogeneses were the risk factors for CAS in Han individuals with $\mathrm{EH}(\mathrm{OR}=1.802)$. MMP-2 CT+TT and MMP-3 6A homogeneses had a combined influence on the incidence of CAS in Han individuals with $\mathrm{EH}$.

Conclusions (1) Han and Uygur individuals had differential distribution of MMPs. (2) The SNP of MMP-2 -735C/T is associated with CAS in individuals with EH. The MMP-2 T allele may be a risk factors on CAS in individuals with EH. The SNP of MMP-3 -1171 $5 \mathrm{~A} / 6 \mathrm{~A}$ is associated with CAS in Han individuals with $\mathrm{EH}$. The $6 \mathrm{~A}$ allele may be a risk factors on CAS in Han individuals with $\mathrm{EH}$.

\section{E0066 ISCHAEMIC PRECONDITIONING COMBINED WITH GRADUAL REPERFUSION OFFERS NO ADDITIONAL BENEFIT ON MITOCHONDRIAL PERMEABILITY PORE OVER PRECONDITIONING OR GRADUAL REPERFUSION ALONE}

doi:10.1136/hrt.2010.208967.66

Dong Chen, Lihuan Li. Fuwai Hospital

Objective Recent investigations demonstrate that ischaemic preconditioning and post-conditioning can reduce infarct size to the same degree. After reflow, opening of the mitochondrial permeability transition pore (mPTP) has been involved in lethal reperfusion injury. We hypothesised that the combination of ischaemic preconditioning and post-conditioning would result in greater 